\title{
Aparelho de avanço mandibular aumenta o volume da via aérea superior de pacientes com apneia do sono
}

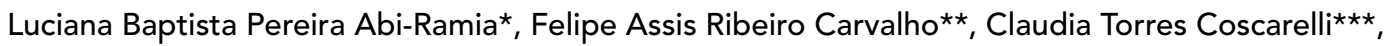

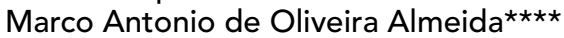

\begin{abstract}
Resumo
Introdução: o diagnóstico, o tratamento e o acompanhamento de pacientes portadores da síndrome da apneia obstrutiva do sono (SAOS) são essenciais, por se tratar de um distúrbio que pode causar alterações sistêmicas. A efetividade do tratamento da SAOS com aparelhos intrabucais foi demonstrada através de estudos cefalométricos. Objetivo: o objetivo deste estudo foi avaliar o efeito do aparelho de avanço mandibular (Twin Block, TB) no volume das vias aéreas superiores, por meio de tomografia computadorizada Cone-Beam (CBCT). Dezesseis pacientes (6 homens e 10 mulheres) portadores de SAOS leve a moderada, idade média de 47,06 anos, utilizaram um aparelho de avanço mandibular e foram acompanhados por 7 meses, em média. Métodos: foram feitas duas CBCT, sendo uma sem e outra com o aparelho em posição. A segmentação e a obtenção dos volumes das vias aéreas superiores foram realizadas e utilizado o teste $t$ de Student pareado para análise estatística, com 5\% de significância. Resultados: houve aumento do volume da via aérea superior com TB quando comparado com o volume sem TB $(p<0,05)$. Conclusão: pode-se concluir que o aumento de volume da via aérea superior observado foi associado ao aparelho de avanço mandibular.
\end{abstract}

Palavras-chave: Síndrome da apneia obstrutiva do sono. Aparelho de avanço mandibular. Tomografia computadorizada de feixe cônico.

\section{INTRODUÇÃO}

Com o aumento das desordens respiratórias do sono, como ronco, síndrome da resistência da via aérea superior (SRVAS) e síndrome da apneia obstrutiva do sono (SAOS), houve a necessidade de melhores meios de diagnóstico e de tratamento para esses distúrbios ${ }^{4,11}$. O tratamento da SAOS é importante ${ }^{11,15,21,25}$, uma vez que é considerada uma doença de alto grau de morbidade e caráter progressivo ${ }^{11,28}$.
A efetividade dos aparelhos de protrusão mandibular foi demonstrada em diversos estu$\operatorname{dos}^{13,25}$. Embora a radiografia cefalométrica seja um método simples, amplamente utilizado na Odontologia e muito usado em estudos de apneia obstrutiva do sono $2,3,4,5,10,13,25,26$, tal método gera imagens bidimensionais de estruturas tridimensionais, o que limita a validade e reprodutibilidade das medidas do espaço aéreo ${ }^{14,16,24}$.

* Mestre em Ortodontia pela Faculdade de Odontologia da Universidade do Estado do Rio de Janeiro (FO-UERJ).

** Doutorando em Ortodontia na FO-UERJ.

*** Especialista e Mestre em Radiologia pela São Leopoldo Mandic.

**** Professor Titular da Disciplina de Ortodontia na FO-UERJ. 
Os estudos baseados em imagens tridimensionais ${ }^{14,21}$ para determinar a efetividade e mecanismo de ação de aparelhos intrabucais demonstraram que o aparelho é capaz de modificar a geometria da faringe $\mathrm{e}^{21}$, aumentando significativamente a área faríngea mínima ${ }^{14}$.

O objetivo deste estudo foi avaliar, através de tomografia computadorizada Cone-Beam (CBCT), o efeito do avanço mandibular por meio do aparelho tipo Twin Block modificado, no volume da via aérea superior de pacientes portadores de SAOS.

\section{MATERIAL E MÉTODOS}

Esta pesquisa foi submetida ao Comitê de Ética do Hospital Universitário Pedro Ernesto, sendo aprovada sob o número 1366-CEP/HUPE.

Os pacientes foram encaminhados para a clínica de pós-graduação da disciplina de Ortodontia da Faculdade de Odontologia da Universidade do Estado do Rio de Janeiro (FO-UERJ) por médicos especialistas em Medicina do Sono, após serem submetidos ao exame de polissonografia noturna para diagnóstico de SAOS leve a moderada $(\mathrm{IAH}<30)$.

Outros critérios de inclusão foram considerados: os pacientes deveriam apresentar índice de massa corporal (IMC) menor do que 27; ter pelo menos dez dentes por arcada, para melhor retenção do aparelho; ter overjet de pelo menos $4 \mathrm{~mm}$, para que o avanço mandibular pudesse ser realizado.

Dezesseis pacientes, 6 homens e 10 mulheres, média de idade de 47,06 anos, receberam aparelhos intrabucais de avanço mandibular do tipo
Twin Block modificado (TB) (Fig. 1), foram orientados a utilizar o aparelho para dormir, e acompanhados por um período médio de 7 meses. $\mathrm{O}$ avanço mandibular proporcionado pelo TB foi de aproximadamente $75 \%$ da protrusão máxima ${ }^{12}$. Para fazer parte da amostra, os pacientes assinaram um termo de consentimento livre e esclarecido, após receberem as informações sobre a pesquisa.

Ao final do período de acompanhamento, cada paciente foi submetido a duas tomografias CBCT (NewTom 3G, Verona, Itália), com campo de visão de 9 polegadas e espessura de corte de $0,2 \mathrm{~mm}$. As tomadas foram realizadas no mesmo dia, sendo uma sem e outra com o aparelho de avanço mandibular. Os pacientes estavam acordados, deitados em decúbito dorsal, com plano de Frankfurt perpendicular ao solo ${ }^{24}$.

A padronização das tomadas foi alcançada com a utilização de um posicionador de acrílico (Fig. 2) e o laser do próprio NewTom 3G, para posicionamento da linha mediana facial. Além disso, as distâncias entre o paciente e o tomógrafo e a altura da maca foram registradas no primeiro exame, para que as duas tomografias fossem o mais semelhante possível. A posição foi conferida no computador através do escanograma, antes do início do segundo exame.

Após a reconstrução primária das projeções nos três planos ortogonais (axial, coronal e sagital), e obtenção das imagens de todo o volume do complexo craniofacial, no formato DICOM (Digital Imaging Communications in Medicine), as imagens foram manipuladas através do software
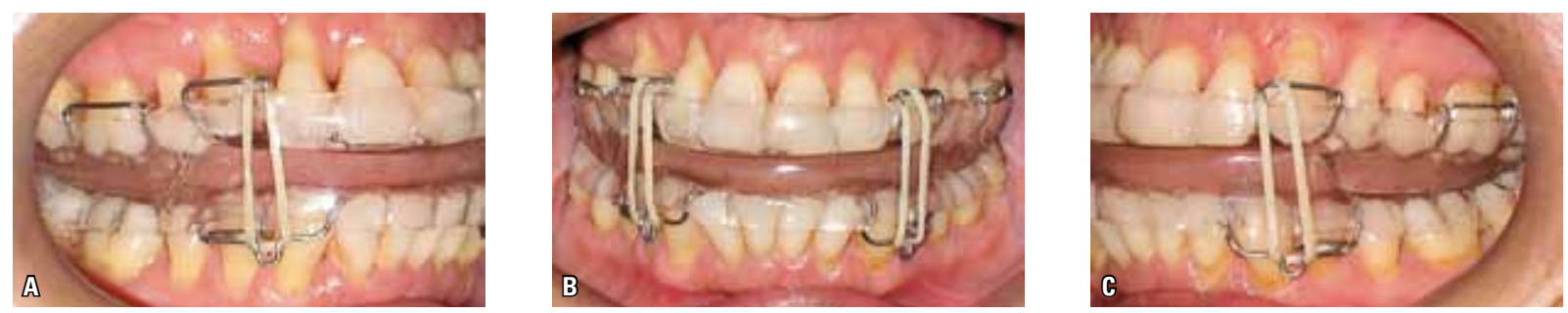

FIGURA 1 - Aparelho Twin Block modificado em posição: A) vista lateral direita, B) vista frontal e C) vista lateral esquerda. 
ITK-SNAP $1.8 .0^{30}$ e obtidas as reconstruções volumétricas da estrutura de interesse. O software permite segmentação semiautomática ${ }^{6,7}$ da área de interesse, que teve como limite anterior e superior a espinha nasal posterior (ENP) ${ }^{24,27}$; e inferior, a região mais anterior e inferior da terceira vértebra cervical (C3) ${ }^{13}$ (Fig. 3). O volume em $\mathrm{mm}^{3}$ do modelo tridimensional da via aérea superior (Fig. 4) foi obtido através do software mencionado.

Os dados estatísticos foram tabulados em um programa estatístico (Biostat 2.0, Belém, Pará, Brasil). O erro de método foi realizado apenas para a delimitação da estrutura, por se tratar de um método semiautomático. Dois avaliadores
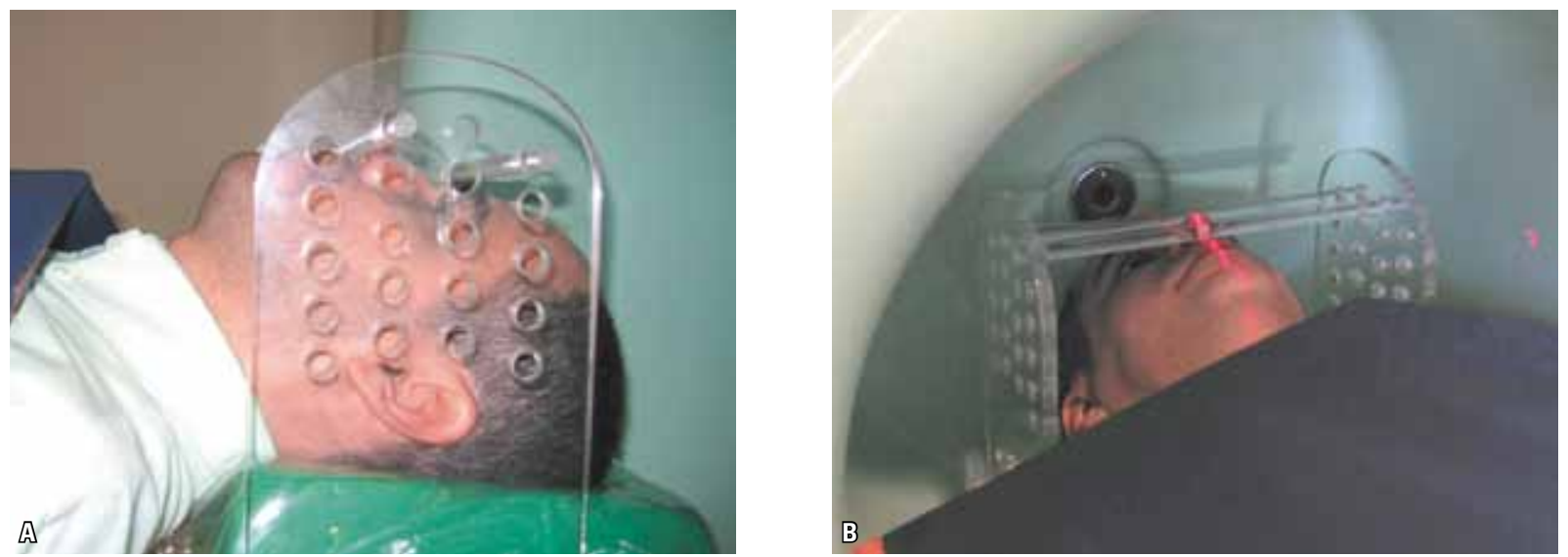

FIGURA 2 - Obtenção da tomografia computadorizada Cone-Beam: A) paciente posicionado no NewTom $3 \mathrm{G}$ com o posicionador de acrílico e com o plano horizontal de Frankfurt perpendicular ao solo; B) laser para posicionamento da linha mediana facial.

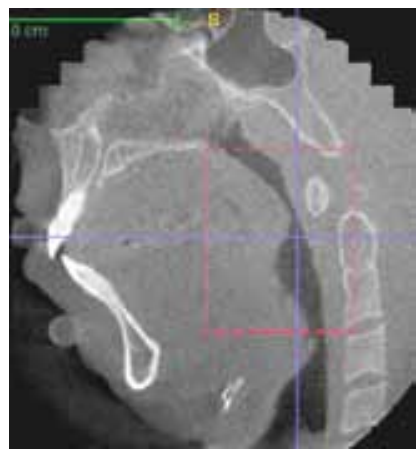

FIGURA 3 - Pontos utilizados para determinar o volume da via aérea superior: ENP (Espinha nasal posterior); C3 (Porção mais anterior e inferior da terceira vértebra). delimitaram a área de interesse duas vezes, com intervalos de dois dias entre elas, e foi utilizado o índice de correlação intraclasse (ICC) para variáveis nominais ou quantitativas, a fim de avaliar a correlação entre medidas repetidas em um mesmo paciente. O ICC demonstrou excelente replicabilidade intraexaminador e interexaminadores, podendo-se afirmar que o método de segmentação e obtenção do volume da via aérea superior é confiável $(\mathrm{p}<0,0001)$.

Após o teste de normalidade Shapiro-Wilk, o teste $t$ pareado foi selecionado para comparação dos volumes sem e com TB. O valor de $\mathrm{p}$ foi estabelecido em 0,05 para ser considerado significativo.

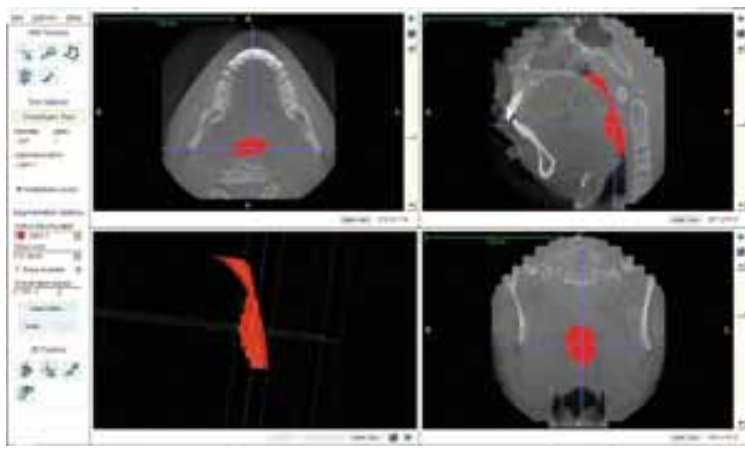

FIGURA 4 - Segmentação do modelo tridimensional. As vias aéreas superiores encontram-se em vermelho. As áreas segmentadas são visualizadas tanto nos cortes tomográficos quanto no modelo tridimensional. 


\section{RESULTADOS}

As médias dos volumes das vias aéreas sem e com TB foram de $7601 \pm 2659 \mathrm{~mm}^{3} \mathrm{e}$ $8710 \pm 2813 \mathrm{~mm}^{3}$, respectivamente (Gráf. 1). Houve diferença estatisticamente significativa $(\mathrm{p}=0,0494)$ entre o volume da via aérea dos pacientes com e sem TB (Tab. 1), demonstrando que o TB foi capaz de aumentar o volume da via aérea superior nos pacientes avaliados.

\section{DISCUSSÃO}

A avaliação tridimensional da via aérea superior foi realizada com CBCT por ser um exame com baixa dose de radiação ${ }^{16,27}$. Segundo Aboudara et al. ${ }^{1}$, apesar de CBCT não ser usualmente indicada para avaliação de tecidos moles, o contraste existente entre a luz da via aérea e os tecidos moles e duros permite o desenvolvimento de uma segmentação acurada para quantificar o volume da via aérea. O NewTom 3G utilizado nesse estudo permitiu a avaliação da via aérea superior enquanto o paciente estava deitado e, apesar de não reproduzir a posição exata do sono, o posicionamento dos tecidos faringeanos é importante para determinar a severidade da síndrome ${ }^{18}$. O posicionamento do paciente durante exames de acompanhamento é muito discutido, uma vez que o fluxo de ar é influenciado por mudanças na posição da cabeça $^{8,29}$, o que pode ser atribuído pela redução da via aérea superior na região retropalatina ${ }^{1}$.

Ao avaliar as imagens nos três planos do espaço, através do software ITK-SNAP, foram escolhidos pontos de referência para delimitação da área de interesse de acordo com estudos prévios ${ }^{24,27}$. Os pontos de referência utilizados nesse estudo foram a ENP1,4,13,24,27 e a porção mais anterior e inferior da terceira vértebra cervical ${ }^{13}$.

A diferença estatisticamente significativa entre o paciente sem e com TB em posição $(p<0,05)$ observada nesse estudo permite afirmar que o aumento da via aérea superior ocorreu em função do avanço mandibular promovido pelo TB. Esse mecanismo ainda está em discussão, no entanto

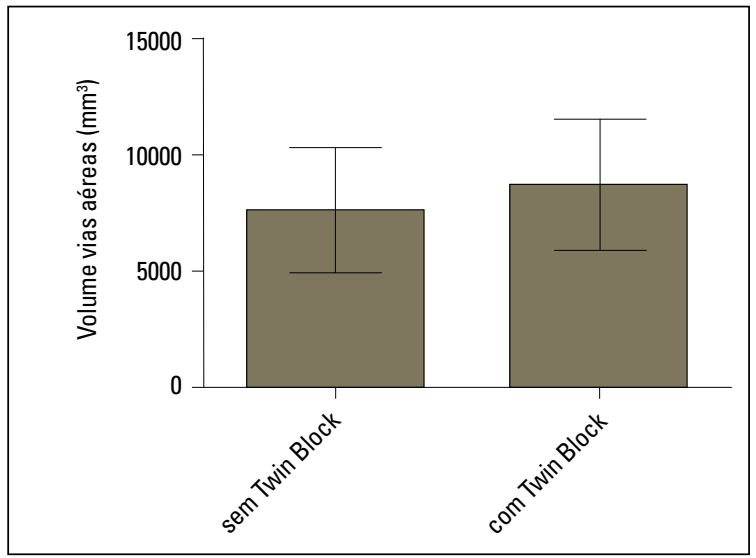

GRÁFICO 1 - Demonstração dos valores do volumes da via aérea superior $\left(\mathrm{mm}^{3}\right)$ dos pacientes sem e com TB.

TABELA 1 - Média, desvio-padrão e valor de p para comparação do volume da via aérea, em $\mathrm{mm}^{3}$, entre os pacientes sem e com TB.

\begin{tabular}{|c|c|c|c|}
\hline & Média & $\begin{array}{l}\text { Desvio- } \\
\text { padrão }\end{array}$ & $\mathbf{p}$ \\
\hline $\begin{array}{l}\text { Volume } \\
\text { sem TB }\end{array}$ & 7601 & 2659 & \multirow{2}{*}{$p=0,0494$} \\
\hline $\begin{array}{l}\text { Volume } \\
\text { com TB }\end{array}$ & 8710 & 2813 & \\
\hline
\end{tabular}

acredita-se que o posicionamento mais anterior da mandíbula e do osso hioide e, consequentemente, a estimulação dos músculos faríngeos e da língua, seja responsável pelo aumento do volume da via aérea ${ }^{30}$. Para Fransson et al. ${ }^{13}$, num estudo cefalométrico, o aumento da área faríngea ocorreu devido ao posicionamento mais anterior do osso hioide pelo aumento da atividade dos músculos genioglosso e pterigóideo lateral.

Apenas dois pacientes apresentaram o volume da via aérea menor com TB do que sem TB, o que pode estar relacionado com a quantidade de avanço mandibular desses pacientes ou com a largura do palato mole. A quantidade de avanço mandibular produzida é muito variável entre os diversos trabalhos, indo desde $2,0 \mathrm{~mm}^{20}$ até $9,5 \mathrm{~mm}^{12,13}$. Apesar das diferenças encontradas, a protrusão de $75 \%$ da capacidade máxima de cada paciente é utilizada com grande concordância por parte dos pesquisadores ${ }^{9,10,13,14,15,17,19,22,23,25}$, 
avanço esse capaz de obter bons índices de sucesso com a terapia e que pode ser suportado pela maior parte dos pacientes.

A comparação, usando CBCT, de pacientes saudáveis com pacientes portadores de SAOS demonstrou que as dimensões anteroposteriores e a área orofaríngea mínima dos pacientes com SAOS foram significativamente menores em relação aos pacientes que não apresentavam a síndrome ${ }^{24}$.

Os estudos tridimensionais em pacientes com SAOS $^{14,21,30}$ demonstram um aumento da via aérea superior, principalmente relacionado à região $\mathrm{da}$ orofaringe $\mathrm{e}^{21}$ e velofaringe $\mathrm{e}^{30}$. Porém, a maioria desses estudos ${ }^{21,30}$ se limitou a avaliar a via aérea a partir de medidas lineares, ou seja, utilizaram dados bidimensionais obtidos através de exames tridimensionais. Segundo Zhao, Liu, Gao ${ }^{30}$ e Kyung, Park, $\mathrm{Pae}^{21}$, o aumento da via aérea ocorre às custas de um incremento do diâmetro transverso. Para Gale et al. ${ }^{14}$, há um aumento da área faríngea com aparelho de avanço mandibular, porém, com uma grande variabilidade individual.

Optou-se pela realização de duas tomografias CBCT no mesmo dia, após o período de acompanhamento, devido à padronização da aquisição da imagem, uma vez que cada paciente tem uma posição ideal diferente. Além disso, poderia haver modificações de IMC e da condição de saúde do paciente durante o acompanhamento, além de mudanças de clima; fatores que invibializariam as comparações entre os tecidos moles e os volumes das vias aéreas superiores em tempos diferentes.

Os estudos com tomografias computadorizadas de feixe cônico e modelos tridimensionais relacionados à SAOS necessitam de mais investigação para melhor padronização dos métodos de avaliação, e também uma melhor compreensão dos mecanismos de ação dos aparelhos de avanço mandibular e seus resultados, a fim de que esses aparelhos possam ser estabelecidos como tratamento de escolha para os pacientes portadores da SAOS.

\section{CONCLUSÕES}

Com base nos resultados apresentados das comparações de volumes em $\mathrm{mm}^{3}$ das vias aéreas superiores de pacientes portadores de SAOS e tratados com aparelho de avanço mandibular, podese concluir que o Twin Block modificado alterou significativamente o volume da via aérea superior.

\title{
Increase in upper airway volume in patients with obstructive sleep apnea using a mandibular advancement device
}

\begin{abstract}
Introduction: Diagnosis, treatment and monitoring of patients with obstructive sleep apnea syndrome (OSAS) are crucial because this disorder can cause systemic changes. The effectiveness of OSAS treatment with intraoral devices has been demonstrated through cephalometric studies. Objective: The purpose of this study was to evaluate the effect of a mandibular advancement device (Twin Block, TB) on the volume of the upper airways by means of Cone-Beam Computed Tomography (CBCT). Sixteen patients ( 6 men and 10 women) with mild to moderate OSAS, mean age 47.06 years, wore a mandibular advancement device and were followed up for seven months on average. Methods: Two CBCT scans were obtained: one with and one without the device in place. Upper airway volumes were segmented and obtained using Student's paired t-tests for statistical analysis with $5 \%$ significance level. Results: TB use increased the volume of the upper airways when compared with the volume attained without TB $(p<0.05)$. Conclusion: It can be concluded that this increased upper airway volume is associated with the use of the TB mandibular advancement device.
\end{abstract}

Keywords: Obstructive sleep apnea syndrome. Mandibular advancement device. Cone-Beam Computed Tomography. 


\section{REFERÊNCIAS}

1. Aboudara C, Nielsen I, Huang JC, Maki K, Miller AJ, Hatcher D. Comparison of airway space with conventional lateral headfilms and 3-dimensional reconstruction from cone-beam computed tomography. Am J Orthod Dentofacial Orthop. 2009 Apr;135(4):468-79.

2. Almeida FR, Lowe AA, Sung JO, Tsuiki S, Otsuka R. Longterm sequellae of oral appliance therapy in obstructive sleep apnea patients: Part 1. Cephalometric analysis. Am J Orthod Dentofacial Orthop. 2006 Feb;129(2):195-204.

3. Battagel JM, Johal A, Kotecha B. A cephalometric comparison of subjects with snoring and obstructive sleep apnoea. Eur $J$ Orthod. 2000 Aug;22(4):353-65

4. Blanco J, Zamarrón C, Abeleira Pazos MT, Lamela C, Suarez Quintanilla D. Prospective evaluation of an oral appliance in the treatment of obstructive sleep apnea syndrome. Sleep Breath. 2005 Mar;9(1):20-5.

5. Cevidanes LH, Styner MA, Proffit WR. Image analysis and superimposition of 3-dimensional cone-beam computed tomography models. Am J Orthod Dentofacial Orthop. 2006 May;129(5):611-8

6. Cevidanes LH, Bailey LJ, Tucker GR Jr, Styner MA, Mol A, Phillips CL, et al. Superimposition of 3D cone-beam CT models of orthognathic surgery patients. Dentomaxillofac Radiol. 2005 Nov;34(6):369-75.

7. Choi JK, Goldman M, Koyal S, Clark G. Effect of jaw and head position on airway resistance in obstructive sleep apnea. Sleep Breath. 2000:4(4):163-8.

8. Clark GT, Arand D, Chung E, Tong D. Effect of anterior mandibular positioning on obstructive sleep apnea. Am Rev Respir Dis. 1993 Mar;147(3):624-9.

9. Cooke ME, Battagel JM. A thermoplastic mandibular advancement device for the management of non-apnoeic snoring: a randomized controlled trial. Eur J Orthod. 2006 Aug;28(4):327-38.

10. Dal Fabbro C, Chaves C Jr, Tufik S. A Odontologia na medicina do sono. $1^{\text {a }}$ ed. Maringá: Dental Press; 2010.

11. Ferguson KA, Ono T, Lowe AA, al-Majed S, Love LL, Fleetham JA. A short term controlled trial of an adjustable oral appliance for the treatment of mild to moderate obstructive sleep apnoea. Thorax. 1997 Apr;52(4):362-8.

12. Fransson AM, Tegelberg A, Svenson BA, Lennartsson B, Isacsson G. Influence of mandibular protruding device on airway passages and dentofacial characteristics in obstructive sleep apnea and snoring. Am J Orthod Dentofacial Orthop. 2002 Oct;122(4):371-9.

13. Fransson AM, Tegelberg A, Johansson A, Wenneberg B. Influence on the masticatory system in treatment of obstructive sleep apnea and snoring with a mandibular protruding device: a 2-year follow-up. Am J Orthod Dentofacial Orthop. 2004 Dec;126(6):687-93.

14. Gale DJ, Sawyer RH, Woodcock A, Stone P, Thompson R, O'Brien K. Do oral appliances enlarge the airway in patients with obstructive sleep apnoea? A prospective computerized tomographic study. Eur J Orthod. 2000 Apr;22(2):159-68.

15. Garib DG, Raymundo R Jr, Raymundo MV, Raymundo DV, Ferreira SN. Tomografia computadorizada de feixe cônico (Cone Beam): entendendo este novo método de diagnóstico por imagem com promissora aplicabilidade na Ortodontia. Rev Dental Press Ortod Ortop Facial. 2007 mar-abr; 12(2):139-56.

16. Horiuchi A, Suzuki M, Ookubo M, Ikeda K, Mitani H, Sugawara J. Measurement techniques predicting the effectiveness of an oral appliance for obstructive sleep apnea hypopnea syndrome. Angle Orthod. 2005 Nov;75(6):1003-11.

17. Ingman T, Nieminen T, Hurmerinta K. Cephalometric comparison of pharyngeal changes in subjects with upper airway resistance syndrome or obstructive sleep apnoea in upright and supine positions. Eur J Orthod. 2004 Jun;26(3):321-6.
18. Johnston CD, Gleadhill IC, Cinnamond MJ, Peden WM Oral appliances for the management of severe snoring: a randomized controlled trial. Eur J Orthod. 2001 Apr;23(2):127-34

19. Jureyda S, Shucard DW. Obstructive sleep apnea: an overview of the disorder and its consequences. Semin Orthod. 2004 Mar;10(1):63-72

20. Kato J, Isono S, Tanaka A, Watanabe T, Araki D, Tanzawa H, et al. Dose-dependent effects of mandibular advancement on pharyngeal mechanics and nocturnal oxygenation in patients with sleep-disordered breathing. Chest. 2000 Apr;117(4):1065-72.

21. Kyung SH, Park YC, Pae EK. Obstructive sleep apnea patients with the oral appliance experience pharyngeal size and shape changes in three dimensions. Angle Orthod. 2005 Jan;75(1):15-22.

22. Marklund M, Franklin KA, Persson M. Orthodontic side-effects of mandibular advancement devices during treatment of snoring and sleep apnoea. Eur J Orthod. 2001 Apr;23(2):135-44.

23. Ogawa T, Enciso R, Shintaku WH, Clark GT. Evaluation of cross-section airway configuration of obstructive sleep apnea. Oral Surg Oral Med Oral Pathol Oral Radiol Endod. 2007 Jan;103(1):102-8.

24. O'Sullivan RA, Hillman DR, Mateljan R, Pantin C, Finucane KE. Mandibular advancement splint: an appliance to treat snoring and obstructive sleep apnea. Am J Respir Crit Care Med. 1995 Jan;151(1):194-8.

25. Otsuka R, Almeida FR, Lowe AA, Ryan F. A comparison of responders and non-responders to oral appliance therapy for the treatment of obstructive sleep apnea. Am J Orthod Dentofacial Orthop. 2006 Feb;129(2):222-9.

26. Tso HH, Lee JS, Huang JC, Maki K, Hatcher D, Miller AJ. Evaluation of the human airway using cone-beam computerized tomography. Oral Surg Oral Med Oral Pathol Oral Radiol Endod. 2009 Nov;108(5):768-76.

27. Walker-Engström ML, Tegelberg A, Wilhelmsson B, Ringqvist I. Four-year follow-up of treatment with dental appliance or uvupalatopharyngoplasty in patients with obstructive sleep apnea. Chest. 2002 Mar;121(3):739-6.

28. Yildirim N, Fitzpatrick MF, Whyte KF, Jalleh R, Wightman AJ, Douglas NJ. The effect of posture on upper airway dimensions in normal subjects and in patients with the sleep apnea/hypopnea syndrome. Am Rev Respir Dis. 1991 Oct;144(4):845-47.

29. Yushkevich PA, Piven J, Hazlett CHC, Smith CRG, Ho CS, Gee $J C$, et al. User-guided $3 D$ active contour segmentation of anatomical structures: Significantly improved efficiency and reliability. Neuroimage. 2006 Jul;31(3):116-28.

30. Zhao X, Liu Y, Gao Y. Three-dimensional upper-airway changes associated with various amounts of mandibular advancement in awake apnea patients. Am J Orthod Dentofacial Orthop. 2008 May;133(5):661-8

Enviado em: junho de 2010

Revisado e aceito: agosto de 2010

Endereço para correspondência

Luciana Baptista Pereira Abi-Ramia

Rua Franz Weissman, 530, bl. 02/ ap. 305 - Barra da Tijuca

CEP: 22.775-051- Rio de Janeiro/RJ

E-mail: labiramia@yahoo.com.br 